\title{
On Witten's Proof of the Positive Energy Theorem^
}

\author{
Thomas Parker ${ }^{1}$ and Clifford Henry Taubes ${ }^{2} \star \star$ \\ 1 Department of Mathematics, Harvard University, Cambridge, MA 02138, USA \\ 2 Department of Physics, Harvard University, Cambridge, MA 02138, USA
}

\begin{abstract}
This paper gives a mathematically rigorous proof of the positive energy theorem using spinors. This completes and simplifies the original argument presented by Edward Witten. We clarify the geometric aspects of this argument and prove the necessary analytic theorems concerning the relevant Dirac operator.
\end{abstract}

The positive energy theorem in general relativity states that an isolated gravitational system with nonnegative local matter density must have nonnegative total energy, measured at spatial infinity. This was originally conjectured more than twenty years ago by Arnowitt, Deser and Misner [1]. Subsequently, a great many people worked on this problem and proved various special cases. Finally, the generic case was established by Schoen and Yau [10-13]. For a history of the problem, with complete references, we refer the reader to the papers of Geroch [5] and Witten [15].

Recently E. Witten has presented a simple new argument for a proof of the positive energy theorem [15]. However, several points of his argument require justification. This paper gives a complete, rigorous and self-contained proof of the positive energy theorem, based on Witten's formulation. In addition to supplying the necessary analytic theorems, we present the proof in its geometric context.

The first three sections present the background for Witten's proof in the language of differential geometry. This involves a brief discussion of Dirac spinors the definition of the Dirac operator along a spacelike hypersurface, and a derivation of Witten's formula for the square of this operator. Section four contains the statement of our main result: the existence of a Green's function for the hypersurface Dirac operator. The positive energy theorem is then proved as a consequence of this fact. The estimates and analysis required for the construction of the Green's function are presented in Sect. five.

* Supported in part by the National Science Foundation under Grant PHY79-16812

$\star \star$ Junior Fellow, Harvard University Society of Fellows 
Estimates similar to those of Sect. 5 have been independently proven by Choquet-Bruhat and Christodoulou [4].

\section{Introduction}

The positive energy theorem, as usually formulated, is a theorem in four-dimensional Lorentzian geometry. We are given a four-manifold $N$ with a metric $g$ of signature $(-+++)$ and a symmetric tensor field $T_{\alpha \beta}$ (interpreted physically as the energy-momentum tensor of matter) which is related to the Ricci and scalar curvatures of $N$ by Einstein's equations

$$
R_{\alpha \beta}-\frac{1}{2} g_{\alpha \beta} R=8 \pi G T_{\alpha \beta}
$$

( $G$ is a constant). We are also given a complete oriented 3-dimensional spacelike hypersurface $M \subset N$ which satisfies the following two conditions:

(i) $M$ is asymptotically flat; there is a compact set $K \subset M$ such that $M-K$ is the disjoint union of a finite number of subsets $M_{1}, \ldots, M_{k}$-called the "ends" of $M$ - each diffeomorphic to the complement of a contractible compact set in $\mathbb{R}^{3}$. Under this diffeomorphism the metric of $M_{l} \subset N$ should be of the form

$$
g_{i j}=\delta_{i j}+a_{i j}
$$

in the standard coordinates $\left\{x^{i}\right\}$ on $\mathbb{R}^{3}$, where $a_{i j}=O(1 / r), \partial_{k} a_{i j}=O\left(1 / r^{2}\right)$ and $\partial_{l} \partial_{k} a_{i j}=O\left(1 / r^{3}\right)$. Furthermore, the second fundamental form $h_{i j}$ of $M \subset N$ should satisfy $h_{i j}=O\left(1 / r^{2}\right)$ and $\partial_{k} h_{i j}=O\left(1 / r^{3}\right)$.

We will often identify the end $M_{l} \subset M$ with the corresponding set $M_{l} \subset \mathbb{R}^{3}$.

(ii) $M$ has nonnegative local mass density: for each point $p \in M$ and for each timelike vector $e_{0}$ at $p, T\left(e_{0}, e_{0}\right) \geqq 0$ and $T\left(e_{0}, \cdot\right)$ is a non-spacelike covector. This has the following consequences: if $\left\{e_{\alpha} / \alpha=0,1,2,3\right\}$ is an adapted orthonormal frame field at $p \in M$ with $e_{0}$ normal to $M$ and $e_{1}, e_{2}, e_{3}$ tangent to $M$, then

and

$$
T^{00} \geqq\left|T^{\alpha \beta}\right| \text { for all } 0 \leqq \alpha, \beta \leqq 3
$$

$$
T^{00} \geqq\left(-T_{0 i} T^{0 i}\right)^{1 / 2} .
$$

(Here, and henceforth, repeated indices are summed with Latin indices running from 1 to 3 and greek indices running from 0 to 3.) This condition if often called the dominant energy condition (Hawking and Ellis [6]).

One can also define the total energy and the total momentum of an asymptotically flat manifold. These quantities include contributions (which cannot be defined separately) from both the matter and the gravitational field itself. They are defined in each asymptotic and $M_{l}$ as limits over the spheres $S_{R, l}$ of radius $R$ in $M_{l} \subset \mathbb{R}^{3}$ :

$$
\begin{aligned}
& E_{l}=\lim _{R \rightarrow \infty} \frac{1}{16 \pi G} \int_{S_{R, l}}\left(\partial_{j} g_{i j}-\partial_{i} g_{i j}\right) d \Omega^{i}, \\
& p_{l k}=\lim _{R \rightarrow \infty} \frac{1}{16 \pi G_{S_{R, l}}} \int_{S_{i k}} 2 \cdot\left(h_{i k}-\delta_{i j} h_{j j}\right) d \Omega^{i} .
\end{aligned}
$$


Remark. There are a number of different expressions for the total momentum $P_{k}$ in the literature. The one above, which is due to Arnowitt, Deser and Misner [1], has the advantage of being defined solely in terms of the data on $M$. The equivalence of the various definitions of $P_{k}$ is discussed in [2].

Physically, a gravitational system with nonnegative matter density should have nonnegative total energy. However, in this mathematical formulation it is not clear that condition (ii) implies anything about the integrals (1.1). The intuition is upheld by the positive energy theorem.

Positive Energy Theorem. Under conditions (i) and (ii) above, $E_{l}-\left|P_{l}\right| \geqq 0$ on each end $M_{l}$. If $E_{l}=0$ for some $l$ then $M$ has only one end and $N$ is flat along $M$.

This is proven in Sect. 4 below.

Remark. If the dominant energy condition holds in a neighborhood of $M$ then $N$ is flat in this neighborhood. This follows by perturbing $M$ in a compact region and applying the above theorem.

There is an important special case. If one further assumes that the metric on $M$ has the asymptotic form

$$
g_{i j}=\left(1+\frac{m_{l}}{2 r}\right)^{4} \delta_{i j}+p_{i j}
$$

in the end $M_{l^{\prime}}$ with $p_{i j}=O\left(1 / r^{2}\right), \partial_{k} p_{i j}=O\left(1 / r^{3}\right)$ and $\partial_{m} \partial_{k} p_{i j}=O\left(1 / r^{4}\right)$, then the positive energy theorem is equivalent to the

Positive Mass Theorem. Assume conditions (i), (ii) and (1.2). Then $m_{l} \geqq 0$ for each $l$, with equality if and only if $M$ is flat along $N$.

\section{Spinors}

Witten's proof depends in an essential way on the use of spinors. Thus we begin with a review of Dirac spinors, describing them first at the level of linear algebra and then globally on the manifold $M$.

Spinors are defined in terms of the representations of $\operatorname{SL}(2, \mathbb{C})$, which is the universal covering group of the connected Lorentz group $\mathrm{SO}(3,1)$. Let $V$ denote the fundamental representation of $\operatorname{SL}(2, \mathbb{C})$ on $\mathbb{C}^{2}$. This representation carries an invariant symplectic form $\sigma$, but has no invariant hermitian structure.

Minkowski space $\mathbb{R}^{3,1}$ is a subspace of $\bar{V} \otimes V$. To see this, consider each $x \in \bar{V} \otimes V$ as a linear transformation $x: V^{*} \rightarrow \bar{V}$ and note that its conjugate transpose is also a map $\bar{x}^{*}: V^{*} \rightarrow \bar{V}$. The fixed set of the involution $x \rightarrow \bar{x}^{*}$ is a real 4-dimensional invariant subspace $W$ of $\bar{V} \otimes V$. The invariant norm on $W$ is, up to a constant, simply the determinant of the transformation $x$. Choosing a basis, we have

$$
\|x\|^{2}=-\operatorname{det} x=-\operatorname{det}\left(\begin{array}{ll}
x_{0}+x_{1} & x_{2}+i x_{3} \\
x_{2}-i x_{3} & x_{0}-x_{1}
\end{array}\right)=-x_{0}^{2}+x_{1}^{2}+x_{2}^{2}+x_{3}^{2},
$$

so $W=\mathbb{R}^{3,1}$. 
Dirac spinors transform according to the representation $S=\bar{V} \oplus V^{*}$. This space has an $\operatorname{SL}(2, \mathbb{C})$ invariant hermitian inner product defined by

$$
(\phi, \psi)=\bar{\xi}_{1} \cdot \eta_{2}+\xi_{2} \cdot \bar{\eta}_{1}
$$

for $\phi=\left(\xi_{1}, \eta_{1}\right) \in S$ and $\psi=\left(\xi_{2}, \eta_{2}\right) \in S$. This inner product is not positive definite.

In this context the Clifford multiplication map $\mathbb{R}^{3,1} \otimes S \rightarrow S$ (denoted by a lower dot $)$ is given by $x \cdot(\xi, \eta)=\left(x \eta, x^{\sigma} \xi\right)$ where $x \in \mathbb{R}^{3,1}$ and $x^{\sigma}: \bar{V} \rightarrow V^{*}$ is the $\sigma$-adjoint of $x: V^{*} \rightarrow \bar{V}$. It follows that $(x . \phi, \psi)=(\phi, x . \psi)$, that is $x$. is a hermitian operator on $S$. To verify the Clifford relations, apply $x$. twice: $x . x .(\xi, \eta)=\left(x x^{\sigma} \xi, x^{\sigma} x \eta\right)$. Since $V^{*}$ is 2-dimensional and $\sigma\left(v^{*},\left(x^{\sigma} x\right) v^{*}\right)=\sigma\left(x v^{*}, x v^{*}\right)=0$ for all $v^{*} \in V^{*}$, we have $x^{\sigma} x=\beta \cdot$ Id. for some $\beta \in \mathbb{C}$. 'Taking the determinant shows that $\beta^{2}=\operatorname{det} x^{\sigma} x=(\operatorname{det} x)^{2}$, so $\beta= \pm\|x\|^{2}$. The correct sign is minus because we can take $x$ to be the identity matrix in (2.1). Thus $x . x .=-\|x\|^{2}$. Id and, by polarization.

$$
x \cdot y .+y \cdot x .=-2 g(x, y) \cdot \mathrm{Id},
$$

where $g$ is the inner product on $\mathbb{R}^{3,1}$.

The choice of a timelike covector $e^{0}$ yields a diagram

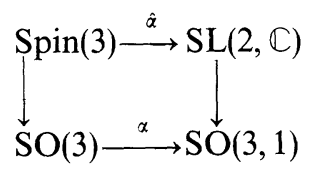

( $\alpha$ and $\hat{\alpha}$ are inclusions) and allows us to regard $V$ as a $\operatorname{Spin}(3)=\operatorname{SU}(2)$ representation. This gives $V$ a hermitian structure, which can be thought of either as the isomorphism $V^{*} \simeq \bar{V}$ given by Clifford multiplication $e^{0} .: V^{*} \rightarrow \bar{V}$, or as a second hermitian inner product on $S$ defined by $\langle\phi, \psi\rangle=\left(e^{0} \cdot \phi, \psi\right)$. This new inner product is $\operatorname{Spin}(3)$ invariant, and under it $e^{0}$. is hermitian and $x$. is skew hermitian whenever $g\left(x, e^{0}\right)=0$. Most importantly, $\langle$,$\rangle is positive definite: in a basis in which$ $e^{0} \cdot: V^{0} \rightarrow \bar{V}$ appears as the identity matrix we have, for $\psi=(\xi, \eta) \in S$,

$$
\langle\psi, \psi\rangle=\left(e_{0} \cdot \psi, \psi\right)=((\eta, \xi),(\xi, \eta))=\bar{\eta} \eta+\xi \bar{\xi} \geqq 0 .
$$

In the physics literature the inner product $(\psi, \psi)$ is denoted $\bar{\psi} \psi=\psi^{+} \gamma^{0} \psi$ and $\langle\psi, \psi\rangle$ is denoted $\psi^{+} \psi$.

These algebraic facts carry over to vector bundles once a spin structure is chosen. Let $F(N)$ denote the $\mathrm{SO}(3,1)$ frame bundle of the cotangent bundle of $N$ and let $i: M \rightarrow N$ be the inclusion. The required spin structure is a lift of the bundle $i^{*} F(N)$ to an $\operatorname{SL}(2, \mathbb{C})$ bundle over $M$. But $i^{*} F(N)=F(M) \times{ }_{\alpha} \mathrm{SO}(3,1)$, so we need only lift the $\mathrm{SO}(3)$ frame bundle of $M$ to a $\operatorname{Spin}(3)$ bundle $F(M)$. The obstruction to such an $\tilde{F}(M)$ is the Stiefel-Whitney class $w_{2}(M)$; this vanishes for orientable 3manifolds. The number of such lifts $\widetilde{F}(M)$ is then classified by $H^{1}\left(M ; \mathbb{Z}_{2}\right)$. Choosing one, we obtain the desired $\operatorname{SL}(2, \mathbb{C})$ bundle $i^{*} F(N)=F(M) \times{ }_{\hat{\alpha}} \operatorname{SL}(2, \overline{\mathbb{C}})$ over $M$ and the associated spin vector bundle

$$
\overparen{i^{*} F(N)} \times{ }_{\rho} S=\tilde{F}(M) \times{ }_{\bar{\rho}} S,
$$

where $\rho$ is the representation $\bar{V} \oplus V^{*}$ of $\operatorname{SL}(2, \mathbb{C})$, and $\bar{\rho}$ is its restriction to $\operatorname{Spin}(3)$. 
This vector bundle-also denoted $S$-carries the inner products (, ) and $\langle$,$\rangle .$ Sections of $S$ are called Dirac spinors along $M$.

The metric connection on $F(N)$ determines connections on $i^{*} F(N)$ and its associated bundles; the resulting connection $\nabla$ on $S$ is compatible with the metric (,) but not compatible with the metrix $\langle$, $\rangle$. On the other hand, the Riemannian connection $\bar{\nabla}$ of $M$ induces a connection on $S=\tilde{F}(M) \times{ }_{\rho} S$ which is compatible with $\langle$,$\rangle but not with ($,$) .$

Finally, we examine the spin structure on the asymptotic ends $M_{l} \subset M$. Let $\Phi_{l}: \mathbb{R}^{3}-K_{l} \rightarrow M_{l}$ be the diffeomorphism which defines $M_{l}$. The pullback bundle $\Phi_{l}^{*} F(M)$ differs from the trivial spin bundle over $\mathbb{R}^{3}-K_{l}$ by an element of $H^{1}\left(\mathbb{R}^{3}-K_{l} ; \mathbb{Z}_{2}\right)=0$. Hence the spin structure is trivial over the ends $M_{l}$ and the bundle $\Phi_{l}^{*} S$ extends trivially over all of $\mathbb{R}^{3}$. The $\Phi_{l}^{-1}$-pullbacks of the constant sections of the bundle $\mathbb{R}^{3} \times S$ over $\mathbb{R}^{3}$ then provide a distinguished set of "constant spinors" over the ends $M_{l}$. These constant spinors will play an important role in Sect. 4 and 5 .

\section{The Hypersurface Dirac Operator}

The Dirac spin bundle $S$ along $M$ is associated to the Spin(3) bundle $\tilde{F}(M)$ and has, in addition to its Riemannian connection $\bar{\nabla}$, the connection $\nabla=\nabla^{N}$ inherited from the four-manifold $N$. We can then define a Dirac operator whose symbol is that of the usual Dirac operator on $M$, but which uses this second connection $\nabla$. We call this the hypersurface Dirac operator and denote it by $\mathscr{D}$. Intrinsically, $\mathscr{D}$ is the composition

$$
\Gamma(S) \stackrel{\nabla}{\rightarrow} \Gamma\left(T^{*} M \otimes S\right) \stackrel{c}{\rightarrow} \Gamma(S)
$$

where $c$ is Clifford multiplication. In a local orthonormal coframe $\left\{e^{i}\right\}$ of $M$

$$
\mathscr{D} \psi=\sum_{i=1}^{3} e^{i} \cdot \nabla_{i} \psi
$$

for $\psi \in \Gamma(S)$.

We are going to derive an equation - a "Weitzenböck formula" - relating the square of $\mathscr{D}$ to the covariant Laplacian. The second fundamental form of the hypersurface $M$ will appear several times in this derivation; it is crucial to keep track of these terms.

Weitzenböck Formula.

$$
\mathscr{D}^{*} \mathscr{D}=\mathscr{D}^{2}=\nabla^{*} \nabla+\mathscr{R}
$$

where $\mathscr{D}^{*}$ and $\nabla^{*}$ are the formal adjoints of $\mathscr{D}$ and $\nabla$ under the inner product $\langle$, and $\mathscr{R}=1 / 4\left(R+2 R_{00}+2 R_{0 i} e^{0} . e^{i}.\right) \in \operatorname{End}(S)$. The integral form of (3.1) is

$$
\begin{aligned}
\int_{M}|\nabla \psi|^{2}+\langle\psi, \mathscr{K} \cdot \psi\rangle-|\mathscr{D} \psi|^{2} & \left.=-\frac{1}{2} \int_{M} d\left[\left\langle\psi,\left[e^{i}, e^{j}\right] \cdot \nabla_{j} \psi\right\rangle e_{i}\right\lrcorner \mu\right] \\
& \left.=-\frac{1}{2} \int_{\partial M}\left\langle\psi,\left[e^{i}, e^{j}\right] \cdot \nabla_{j} \psi\right\rangle e_{i}\right\lrcorner \mu
\end{aligned}
$$


for any Dirac spinor $\psi$ along $M$, where $\left[e^{i}, e^{j}\right]=e^{i} \cdot e^{j}-e^{j} \cdot e^{i}$ is the commutator in the Clifford algebra.

Remark. The endomorphism $\mathscr{R}$ can be written in terms of $T_{\alpha \beta}$ by Einstein's equations; in fact $\mathscr{R}=4 \pi G\left(T_{00}+T_{0 i} e^{0} . e^{i}.\right)$. The dominant energy condition then implies that $\mathscr{R} \geqq 4 \pi G\left[T_{00}-\left(-T_{0 i} T^{0 i}\right)^{1 / 2}\right] \geqq 0$.

The verification of (3.1) and (3.2) is simpler when done in the moving frame defined as follows. Fix a point $p \in M$ and an orthonormal basis $\left\{e_{\alpha}\right\}$ of $T_{p} N$ with $e_{0}$ normal and $e_{1}, e_{2}, e_{3}$ tangent to $M$. Extend $\left\{e_{1}, e_{2}, e_{3}\right\}$ to an orthonormal frame in a neighborhood of $p$ in $M$ in such a way that $\left(\bar{\nabla}_{i} e_{j}\right)_{p}=0$ for $1 \leqq i, j \leqq 3$. Extend this to a local orthonormal frame $\left\{e_{\alpha}\right\}$ for $N$ with $\left(\nabla_{0} e_{i}\right)_{p}=0$ for $i=1,2,3$. Let $\left\{e^{\alpha}\right\}$ be the dual coframe. Then $\left(\nabla_{i} e^{J}\right)_{p}=-h_{i j} e^{0}$ and $\left(\nabla_{i} e^{0}\right)_{p}=-h_{i j} e^{J} \quad$ where $h_{i j}=\left\langle\nabla_{e_{t}} e_{0}, e_{j}\right\rangle$ are the components of the second fundamental form at $p$.

In this frame we have, at $p \in M$,

$$
\begin{aligned}
\mathscr{D}^{2} & =\sum_{i, j} e^{i} \cdot \nabla_{i}\left(e^{j} \cdot \nabla_{j}\right)=\sum_{i, j} e^{i} \cdot e^{j} \cdot \nabla_{i} \nabla_{j}+\sum_{i, j} e^{i} \cdot\left(\nabla_{i} e^{j}\right) \nabla_{j} \\
& =\sum_{i} \nabla_{i} \nabla_{i}+\frac{1}{2} \sum_{i \neq j} e^{i} \cdot e^{j} \cdot\left(\nabla_{i} \nabla_{j}-\nabla_{j} \nabla_{i}\right)-\sum_{i, j} h_{i j} e^{i} \cdot e^{0} \cdot \nabla_{j} .
\end{aligned}
$$

Since $\left[e_{i}, e_{j}\right]_{p}=0$, the middle term is the curvature expression

$$
\frac{1}{2} \Omega_{i j} e^{i} \cdot e^{j} .=\frac{1}{4} R_{\alpha \beta i j} e^{i} \cdot e^{j} \otimes \rho\left(e^{\alpha} \wedge e^{\beta}\right),
$$

where $\rho: s o(3,1) \rightarrow \operatorname{End}(S)$ is the spin representation, given by $\rho\left(e^{\alpha} \wedge e^{\beta}\right)=$ $-1 / 2 e^{\alpha} \cdot e^{\beta}$. This is easily simplified using the Bianchi identity and the symmetries of $R$. Equation (3.1) then reads

$$
\mathscr{D}^{2}=-\nabla_{i} \nabla_{i}+\frac{1}{4}\left(R+2 R_{00}+2 R_{0 j} e^{0} \cdot e^{j} \cdot\right)-h_{i j} e^{i} \cdot e^{0} \cdot \nabla_{j} .
$$

Next, we need the integration by parts formulae for $\nabla$ and $\mathscr{D}$ with respect to the positive definite inner product $\langle$,$\rangle . Again computing in our local frame at p$,

$$
\begin{aligned}
d\left[\left\langle\phi, \nabla_{i} \psi\right\rangle e_{i}-\mu\right] & =\left[\left(\left(\nabla_{i} e^{0}\right) \cdot \phi, \nabla_{i} \psi\right)+\left(e^{0} \cdot \nabla_{i} \phi, \nabla_{i} \psi\right)+\left(e^{0} \cdot \phi, \nabla_{i} \nabla_{i} \psi\right)\right] \mu \\
& =\left[\left(-h_{i j} e^{J} \cdot \phi, \nabla_{i} \psi\right)+\left\langle\nabla_{i} \phi, \nabla_{i} \psi\right\rangle+\left\langle\phi, \nabla_{i} \nabla_{i} \psi\right\rangle\right] \mu \\
& =\left[\left\langle\phi, h_{i j} e^{l} \cdot e^{0} \cdot \nabla_{j} \psi\right\rangle+\left\langle\nabla_{i} \phi, \nabla_{i} \psi\right\rangle+\left\langle\phi, \nabla_{i} \nabla_{i} \psi\right\rangle\right] \mu
\end{aligned}
$$

where $\mu=e^{1} \wedge e^{2} \wedge e^{3}$ is the volume form on $M$ and $\lrcorner$ denotes contraction. In particular, this shows that the formal $\langle$,$\rangle -adjoint of \nabla$ is given by $\nabla_{i}^{*}=-\nabla_{i}$ $-h_{i j} e^{j} \cdot e^{0}$.

A similar calculation yields

$$
\left.d\left[\left\langle\phi, e^{i} . \psi\right\rangle e_{j}\right\lrcorner \mu\right]=[\langle\phi, \mathscr{D} \psi\rangle-\langle\mathscr{D} \phi, \psi\rangle] \mu,
$$

so $\mathscr{D}$ is formally self-adjoint under $\langle$,$\rangle . Together (3.4), (3.5) and (3.6) give (3.1),$ which is valid in any frame.

The integral expression (3.2) follows by applying (3.1) to $\psi$, taking the inner product with $\psi$, and integrating by parts. The boundary term, which arises from (3.5) and (3.6), has been simplified using

$$
\nabla_{i} \psi+e^{i} \cdot \mathscr{D} \psi=\left(\delta^{i j}+e^{i} \cdot e^{j} \cdot\right) \nabla_{j} \psi=+1 / 2\left[e^{i}, e^{j}\right] \cdot \nabla_{j} \psi .
$$




\section{The Positive Energy Theorem}

The Weitzenbock formula (3.2) and the remark which follows it lead to the vanishing theorem discovered by Witten: under the conditions of the positive energy theorem, if $\psi$ is a spinor field along $M$ which satisfies $\mathscr{D} \psi=0$ and which vanishes at infinity fast enough that the boundary term in (3.2) is zero, then $\psi \equiv 0$. Furthermore, if $\psi$ is an asymptotically constant solution of $\mathscr{D} \psi=0$ then the left-hand side of (3.2) is positive while the boundary term is essentially the total energy-momentum of $M$. In this sense the positive energy theorem is a consequence of formula (3.2).

In this section we will prove the positive energy theorem by this argument. The key step in the proof is the construction of a Green's function for the operator $\mathscr{D}$; this is carried out in Theorems 4.1 and 4.3. There are several features of this argument which should be emphasized.

The first concerns the proof that if $\mathscr{D} \psi=0$ and $\psi$ vanishes at infinity then $\psi$ vanishes identically. These hypotheses and Weitzenböck formula (3.2) imply that $\nabla \psi=0$, but, since $\nabla$ is not compatible with the inner product, it does not follow immediately that $\psi$ vanishes. In fact the argument (Lemma 4.3) depends crucially on the decay of the second fundamental form. Second, the fact that the boundary term of (3.2) reduces to the total energy-momentum of $M$ is actually quite general: it is true for any $\psi$ which approaches an asymptotically constant spinor sufficiently fast, independent of whether $\mathscr{D} \psi=0$. Finally, to prove "if the spacelike hypersurface $M$ has zero energy then $N$ is flat along $M$ ", it is not necessary to perturb the hypersurface (as Witten does); the conclusion follows directly from the dominant energy condition.

Throughout this section we will assume that $M$ and $N$ satisfy conditions (i) and (ii) of Sect. 1. Our first theorem guarantees the existence of harmonic spinors with prescribed asymptotics.

Theorem 4.1. Let $M$ and $N$ be as in Theorem 4.1. Let $\left\{\psi_{0 l}\right\}_{l=1}^{k}$ be constant spinors defined in the asymptotic ends $\left\{M_{l}\right\}_{l=1}^{k}$. Then there exists a unique, smooth spinor $\psi$ on $M$ that satisfies

(i) $\mathscr{D} \psi=0$.

(ii) For every $\varepsilon>0, \lim _{r \rightarrow \infty} r^{1-\varepsilon}\left|\psi-\psi_{0 l}\right|=0$ in each end $M_{l}$.

(iii) In addition,

$$
\begin{aligned}
0 & \leqq \int_{M} \mu\{\langle\nabla \psi, \nabla \psi\rangle+\langle\psi, \mathscr{R} \cdot \psi\rangle\} \\
& =4 \pi G \sum_{l=1}^{k}\left(E_{l}\left\langle\psi_{0 l}, \psi_{0 l}\right\rangle+\left\langle\psi_{0 l}, p_{l k} d x^{0} \cdot d x^{k} \cdot \psi_{0 l}\right\rangle\right) .
\end{aligned}
$$

Here $\left\{d x^{\alpha}\right\}$ is the standard basis of $T^{*}\left(\mathbb{R}^{3,1}\right)$.

Theorem 4.1 follows from a technical result which establishes both the existence of the Green's function for the operator $\mathscr{D}$ and the space of spinors on which this Green's function acts. For this reason, we use weighted Sobolev spaces, which are the natural analogues on asymptotically flat manifolds of the standard $L_{p}^{k}$ spaces on compact manifolds.

Definition 4.1. Let $R \geqq 1$ be large enough so that each end $M_{l} \subset \mathbb{R}^{3}$ contains the 
exterior of the ball $B_{R}$ of radius $R$. For each $l$ and each $r \geqq R$, set $M_{l, r}=M_{l}-B_{r}$, considered either as a subset of $\mathbb{R}^{3}$ or of $M$. Fix a smooth function $\sigma$ on $M$ with the following properties: (i) $\sigma \geqq 1$, (ii) $\sigma=r$ in $M_{l, 2 R}$, and (iii) $\sigma=1$ in $M-\cup_{l} M_{l, R}$.

Definition 4.2. For $p \geqq 2,1 / 2-3 / p \leqq \delta \leqq 2-3 / p$, and $s=0$ or 1 , the Banach space $\mathscr{H}_{s, \delta, p}$ is the completion of $C_{0}^{\infty}(M ; S)$ (smooth sections of the vector bundle $S$ with compact support) in the norm

where

$$
\|\psi\|_{s, \delta, p}=s\left\|\sigma^{1+\delta} \bar{\nabla} \psi\right\|_{p}+\left\|\sigma^{\delta} \psi\right\|_{p},
$$

$$
\|\psi\|_{p}=\left(\int_{M}\langle\psi, \psi\rangle^{p / 2}\right)^{1 / p}
$$

is the $L_{p}$ norm. For notational convenience, denote $\mathscr{H}_{1,-1,2}$ by $\mathscr{H}$ and $\|\cdot\|_{1,-1,2}$ by $\|\cdot\|_{\mathscr{H}}$.

The existence of a Green's function for the operator $\mathscr{D}$ can be stated in terms of these Sobolev spaces as follows.

Theorem 4.2. For $p \geqq 2$ and $0<\delta<2-3 / p$, or for $p=2$ and $\delta=-1$, the operator $\mathscr{D}: \mathscr{H}_{1, \delta, p} \rightarrow \mathscr{H}_{0, \delta+1, p}$ is an isomorphism with bounded inverse $\mathscr{D}^{-1}$. If $\eta \in \mathscr{H}_{0, \delta+1, p} \cap C^{\infty}(M ; S)$ then $\mathscr{D}^{-1} \eta \in \mathscr{H}_{1, \delta, p} \cap C^{\infty}(M ; S)$.

The proof of Theorem 4.2 is presented in the next section. Here we will use it to prove Theorem 4.1 and the positive energy theorem.

Proof of Theorem 4.I. In each end $l$, fix a smooth function $0 \leqq \beta_{R}(l) \leqq 1$ which is identically 1 in the end $M_{l, 3 R}$ and 0 inside $M_{l, 2 R}$. Let $\psi_{0} \in C(M ; S)$ be the spinor

$$
\psi_{0}=\sum_{l=1}^{k} \psi_{0 l} \beta_{R}(l)
$$

The hypotheses imply that $\mathscr{D} \psi_{0}=O\left(r^{-2}\right)$ and hence that $\mathscr{D} \psi_{0} \in L_{2} \cap \mathscr{H}_{0,1+\delta, p}$ for all $2 \leqq p<\infty$ and $0<\delta<1-3 / p$. By Theorem 4.3, there exists a unique spinor, $\psi_{1}$, in $\mathscr{H} \cap \mathscr{H}_{1, \delta, p} \cap C^{\infty}(M ; S)$ such that

$$
\mathscr{D} \psi_{1}=-\mathscr{D} \psi_{0} \text {. }
$$

Hence $\psi=\psi_{0}+\psi_{1}$ is a harmonic spinor. Note that because $\psi_{1} \in \mathscr{H}_{1, \delta, p}$ the function $\sigma^{\delta}\left\langle\psi_{1}, \psi_{1}\right\rangle^{1 / 2} \in L_{p}^{1}(M)$ for all $0<\delta<1-3 / p$ and $2 \leqq p<\infty$. For $3<p<\infty, L_{p}^{1}$ $\hookrightarrow C^{0}$, hence $L_{p}^{1}$ functions have asymptotic decay [9; pg. 79]. This establishes statements i) and ii) of Theorem 4.1.

To establish statement (iii), we utilize the integration by parts formula (3.2). Since $\mathscr{D} \psi=0$, we have

$$
\int_{M}\{\langle\nabla \psi, \nabla \psi\rangle+\langle\psi, \mathscr{R} \cdot \psi\rangle\} \mu=-\int_{M}\left[d \eta+\frac{1}{2} d\left(\left\langle\psi_{0},\left[e^{l}, e^{j}\right] \cdot \nabla_{j} \psi_{0}\right\rangle e_{i}\right\lrcorner \mu\right]
$$

where $\eta$ is the 2 -form

$$
\left.\eta=\frac{1}{2}\left[\left\langle\psi_{1},\left[e^{i}, e^{j}\right] \cdot \nabla_{j} \psi\right\rangle+\left\langle\psi_{0},\left[e^{i}, e^{j}\right] \cdot \nabla_{j} \psi_{1}\right\rangle\right] e_{i}\right\lrcorner \mu .
$$

The left-hand side of (4.2) is real, so the imaginary part of the right side must vanish. Indeed, a direct calculation (best done in the frame used in Sect. 3) shows 
that

$$
\left.\left.d\left[\left\langle\psi_{0},\left[e^{i}, e^{j}\right] \cdot \psi_{1}\right\rangle e_{i}\right\lrcorner \mu\right]=\left[\left\langle\nabla_{j} \psi_{0},\left[e^{i}, e^{j}\right] \cdot \psi_{1}\right\rangle+\left\langle\psi_{0},\left[e^{i}, e^{j}\right] \cdot \nabla_{j} \psi_{1}\right\rangle\right] e_{i}\right\rfloor \mu,
$$

so we can replace $\eta$ in (4.2) by

$$
\left.\hat{\eta}=\frac{1}{2}\left\{\left\langle\psi_{1},\left[e^{i}, e^{j}\right] \cdot \nabla_{j} \psi\right\rangle-\left\langle\nabla_{j} \psi_{0},\left[e^{i}, e^{j}\right] \cdot \psi_{1}\right\rangle\right\} e_{i}\right\lrcorner \mu .
$$

We now observe that $d \hat{\eta} \in L^{1}$. This is seen by differentiating $\hat{\eta}$, using $\nabla_{i}=\bar{\nabla}_{i}-$ $\frac{1}{2} h_{i j} e^{0} \cdot e^{j}$. and $\left[e^{i}, e^{j}\right] \cdot \bar{\nabla}_{i} \bar{\nabla}_{j}=\frac{1}{2}\left[e^{i}, e^{j}\right] \cdot\left(\bar{\Omega}_{i j}+\bar{\nabla}_{\left[e_{i} e_{j}\right]}\right)$ where $\bar{\Omega}_{i j} \in \operatorname{End}(S)$ is the curvature of $M$ :

$$
\begin{aligned}
2 d \hat{\eta}=\{ & \left\langle\bar{\nabla}_{i} \psi_{1},\left[e^{i}, e^{j}\right] \cdot \nabla_{j} \psi\right\rangle+\frac{1}{2}\left\langle\psi_{1},\left[e^{i}, e^{j}\right] \bar{\Omega}_{i j} \cdot \psi\right\rangle-\frac{1}{2}\left\langle\psi_{1},\left[e^{i}, e^{j}\right] \cdot \bar{\nabla}_{i} h_{j k} e^{0} \cdot e^{k} \cdot \psi\right\rangle \\
& \left.-\frac{1}{2}\left\langle\bar{\Omega}_{i j} \cdot \psi_{0},\left[e^{i}, e^{j}\right] \cdot \psi_{1}\right\rangle+\frac{1}{2}\left\langle\bar{\nabla}_{j} h_{i k}\right) e^{0} \cdot e^{k} \cdot \psi_{0},\left[e^{i}, e^{m}\right] \cdot \psi_{1}\right\rangle \\
& \left.-\left\langle\nabla_{j} \psi_{0},\left[e^{i}, e^{j}\right] \cdot \bar{\nabla}_{i} \psi_{i}\right\rangle\right\} .
\end{aligned}
$$

This gives the pointwise estimate

$$
\begin{aligned}
|d \hat{\eta}| \leqq & \left\{\left|\bar{\nabla} \psi_{1}\right|^{2}+\frac{1}{2}|\nabla \psi|^{2}+\left|\psi_{1}\right| \cdot|\psi| \bar{\Omega}|+| \psi_{1}|\cdot| \bar{\nabla} h|\cdot| \psi|+| \psi_{0}|\cdot| \psi_{1}|\cdot| \bar{\Omega} \mid\right. \\
& \left.+|\bar{\nabla} h| \cdot\left|\psi_{0}\right| \cdot\left|\psi_{1}\right|+\frac{1}{2}\left|\nabla \psi_{0}\right|^{2}\right\} .
\end{aligned}
$$

Since $\psi_{1} \in \mathscr{H}$, the spinors $\bar{\nabla} \psi_{1}, h_{i j} \psi_{1}$ and $\sigma^{-1} \psi_{1}$ are square integrable, while $\nabla \psi_{0}$ is square integrable since $M$ is asymptotically flat (cf. Eq. (4.4) below). (Recall that $\bar{\nabla} h$ and $\bar{\Omega}$ are $O\left(1 / r^{3}\right)$. It follows that $d \hat{\eta} \in L^{1}$.

Let $w_{\rho}$ be a cutoff function with (i) $w_{\rho}=0$ on $\cup_{l=1}^{k} M_{l, 2 \rho}$, (ii) $w_{\rho}=1$ on the complement of $\cup_{l=1}^{k} M_{l, \rho}$ in $M$ and (iii) $\left|d w_{\rho}\right|<2 / \rho$.

Since $d \hat{\eta} \in L_{1}$, the dominated convergence theorem implies that,

$$
\begin{aligned}
\int_{M} d \hat{\eta} & =\lim _{\rho \rightarrow \infty} \int_{M} w_{\rho} d \hat{\eta}=\lim _{\rho \rightarrow \infty} \int_{M}\left\{d\left(w_{\rho} \hat{\eta}\right)-d w_{\rho} \wedge \hat{\eta}\right\} \\
& =\lim _{\rho \rightarrow \infty} \int_{M} d w_{\rho} \wedge \hat{\eta} .
\end{aligned}
$$

For sufficiently large $\rho,\left|\sigma \cdot d w_{\rho}\right| \leqq 2, d w_{\rho}=\left(1-w_{\rho / 2}\right) d w_{\rho}$, and

$$
\int\left|d w_{\rho} \wedge \hat{\eta}\right| \mu \leqq 2\left\|\psi_{1} d w_{\rho}\right\|_{2}\left(\|\nabla \psi\|_{2}+\left\|\nabla \psi_{0}\right\|_{2}\right)
$$

$$
\left.\leqq 4\left\|\left.\sigma^{-1} \psi_{1}\left(1-w_{\rho / 2}\right)\right|_{2}+\right\| \nabla \psi_{0} \|_{2}\right) \text {. }
$$

This vanishes as $\rho \rightarrow \infty$. We are left with

$$
\left.\int_{\Psi} \mu\{\langle\nabla \psi, \nabla \psi\rangle+\langle\psi, \mathscr{R} . \psi\rangle\}=\lim _{r \rightarrow \infty}-\frac{1}{2} \sum_{l=1}^{k} \int_{\partial}\left\langle\psi_{l, r},\left[e^{i}, e^{j}\right] \cdot \nabla_{j} \psi_{0}\right\rangle e_{i}\right\rfloor \mu .
$$

The right-hand side of (4.3) explicitly depends only on the asymptotic data.

The proof of statement (iii) is completed by expressing the boundary term of (4.3) in the coordinate system given by the diffeomorphism $\Phi_{l}: \mathbb{R}^{3}-K_{l} \rightarrow M_{l}$ which defines the end $M_{l}$. The hermitian structure $e^{0}$. on $S$ corresponds under $\Phi_{l}$ to the hermitian structure $d x^{0}$. on $\Phi_{l}^{*} S=\mathbb{R}^{3} \times S$ arising from the inclusion $\mathbb{R}^{3} \subset \mathbb{R}^{3,1}$. The expression for the spin connection on $\Phi_{l}^{*} S$ in terms of the metric connection and the second fundamental form is then

$$
\nabla_{j} \psi_{0}=\bar{\nabla}_{j} \psi_{0}-\frac{1}{2} h_{j k} d x^{0} \cdot d x^{k} \cdot \psi_{0}=\partial_{j} \psi_{0}-\frac{1}{4} \Gamma_{k j l} d x^{k} \cdot d x^{l} \cdot \psi_{0}-\frac{1}{2} h_{j k} d x^{0} \cdot d x^{k} \cdot \psi_{0},
$$


where $\Gamma_{k j l}$ are the connection components and $\partial_{j} \psi_{0}=0$ since $\psi_{0}$ is constant. The hypotheses allow for an orthonormal coframe $\left\{e^{i}\right\}$ with $\left|e^{i}-d x^{i}\right|=O(1 / r)$, so we can replace the $\left\{e^{i}\right\}$ by $\left\{d x^{i}\right\}$ in (4.3) without changing the value of the limit. Using (4.4), the symmetry $\Gamma_{k j l}=\Gamma_{k l j}$ and the formula for $\Gamma_{k j l}$ in terms of the metric, we find that the boundary term of (4.3) is

$$
\begin{aligned}
-\frac{1}{2} \int\left\langle\psi_{0},\left[d x^{i}, d x^{j}\right] \cdot \nabla_{j} \psi_{0}\right\rangle d \Omega^{i}= & -\frac{1}{2} \int\left\langle\psi_{0},\left\{-\frac{1}{42} \cdot \Gamma_{k j l}\left[d x^{i}, d x^{j}\right] \cdot\left[d x^{k}, d x^{l}\right]\right.\right. \\
& \left.\left.-\frac{1}{2} h_{j k}\left[d x^{i}, d x^{j}\right] \cdot d x^{0} \cdot d x^{k}\right\} \cdot \psi_{0}\right\rangle d \Omega^{i} \\
= & \frac{1}{4} \int\left\langle\psi_{0},-\frac{1}{4} \Gamma_{k j l} \cdot 4\left(\delta^{i k} \delta^{j l}-\delta^{i l} \delta^{j k}\right) \psi_{0}\right. \\
& \left.+h_{j k} \cdot 2\left(\delta^{i j} d x^{0} \cdot d x^{k}-\delta^{i k} d x^{0} \cdot d x^{i}\right) \cdot \psi_{0}\right\rangle d \Omega^{i} \\
= & \frac{1}{4} \int\left\langle\psi_{0},\left(\partial_{j} g_{i j}-\partial_{i} g_{j j}\right) \psi_{0}+2\left(h_{i k}-\delta_{i k} h_{j j}\right)\right. \\
& \left.\cdot d x^{0} \cdot d x^{k} \cdot \psi_{0}\right\rangle d \Omega^{i} \\
= & 4 \pi G\left\langle\psi_{0}, E \psi_{0}+p_{k} d x^{0} \cdot d x^{k} \cdot \psi_{0}\right\rangle
\end{aligned}
$$

on each end $M_{l}$. Here $\left.d \Omega^{l}=\frac{\partial}{\partial x^{l}}\right\lrcorner \mu$.

Lemma 4.3. Suppose that $\psi$ and $\left\{\psi_{i}\right\}$ are smooth spinors along $M$ with $\nabla \psi=0$ and $\nabla \psi_{i}=0$ for each $i$.

(a) If $\lim \psi(x)=0$, where this limit is taken along some path in one asymptotic end $M_{l}$, then $\psi=0$.

(b) If $\left\{\psi_{i}\right\}$ are linearly independent in some end $M_{l}$, then they are linearly independent everywhere on $M$.

Proof. (a) Set $|\psi|=\langle\psi, \psi\rangle^{1 / 2}$. Calculating in the orthonormal frame used in Sect. 3 we obtain

$$
d|\psi|^{2}=\left[-\left(h_{i j} e^{j} \cdot \psi, \psi\right)+\left\langle\nabla_{i} \psi, \psi\right\rangle+\left\langle\psi, \nabla_{i} \psi\right\rangle\right] e^{i}=-\left(h_{i j} e^{j} \cdot \psi, \psi\right) e^{i}
$$

and the pointwise estimate $2|\psi| \cdot|d| \psi||=\left.\left.|d| \psi\right|^{2}|\leqq| h|\cdot| \psi\right|^{2}$. Since $h=O\left(1 / r^{2}\right)$ this gives $|d \ln | \psi \mid \leqq C / r^{2}$ on the complement of the zero set of $\psi$. Integrating this along a path from $x_{0} \in M$ to $x$ gives $|\psi(x)| \geqq\left|\psi\left(x_{0}\right)\right| \cdot \exp C\left(1 /|x|-1 /\left|x_{0}\right|\right)$, and taking $x$ to be the first zero of $\psi$ along the path of integration, or taking the limit as $|x| \rightarrow \infty$ if no such zero exists, shows that $\psi\left(x_{0}\right)=0$.

(b) Suppose that there are constants $c_{i}$ such that $\psi=\sum c_{l} \psi_{i}$ vanishes at some point $x_{0} \in M$. Since $\nabla \psi=0$ we can repeat the above argument to conclude that $0=\left|\psi\left(x_{0}\right)\right| \geqq|\psi(x)| \exp C \cdot\left(1 /|x|-1 /\left|x_{0}\right|\right)$ for any $x \in M$; this contradicts the hypothesis.

We can now prove the positive energy theorem stated in Sect. 1.

Proof of the Positive Energy Theorem. Let $P_{i, m}, i=1,2,3$ be the components of the total momentum of the end $M_{m} \subset \mathbb{R}^{3}$. Let $\left\{\psi_{m}\right\}_{m=1}^{k}$ be constant spinors on the asymptotic ends with $\psi_{m}=0$ on each end except $M_{l}$, and $\psi_{l}$ an eigenvalue of the hermitian matrix $P_{i l} d x^{0} . d x^{i} . \in \operatorname{End}(S)$ with eigenvalue $-|P|$. Theorem 4.1 then gives a harmonic spinor $\psi$ with asymptotics $\left\{\psi_{m}\right\}$. Substituting $\psi$ into (4.1) shows that $E_{l}-\left|P_{l}\right| \geqq 0$. 
Now suppose that the energy of some end, say $M_{1}$, is zero. Choose a basis $\left\{\Psi^{a} \mid a=1,2,3,4\right\}$ of constant spinors and take as asymptotic data the constant spinors $\left\{\psi_{l}^{a}\right\}$ with $\psi_{l}^{a}=\bar{\psi}^{a}$ on $M_{1}$ and $\psi_{l}^{a}=0$ on all other ends $M_{l}$. Let $\left\{\psi^{a}\right\}$ be the solutions of $\mathscr{D} \psi^{a}=0$ constructed from this data by Theorem 4.1. The boundary term in (4.1) then vanishes, so $\nabla \psi^{a}=0$ and $\psi^{a} \rightarrow 0$ uniformly on each and except $M_{1}$. But this contradicts Lemma $4.3 \mathrm{a}$ unless $M_{1}$ is the only end of $M$.

Because $\left\{\psi^{a}\right\}$ are linearly independent on $M_{1}$ they are linearly independent everywhere by Lemma $4.3 \mathrm{~b}$. Furthermore, $\nabla \psi^{a}=0$, so in a local frame $\left\{e_{i}\right\}$ of $M$,

$$
0=\left(\nabla_{i} \nabla_{j}-\nabla_{j} \nabla_{i}-\nabla_{\left[e_{i} e_{j}\right]}\right) \psi^{a}=-\frac{1}{4} R_{\alpha \beta i j} e^{\alpha} \cdot e^{\beta} \cdot \psi^{a}
$$

for all $1 \leqq i, j \leqq 3$. This implies that $R_{\alpha \beta i j}=0$ because $\left\{\psi^{a}\right\}$ are a basis of $S$ and $S$ is a faithful representation of $\lrcorner \ell(2, C)$. Subsequently, $T_{\alpha \beta} \equiv 0$ : by Einstein's equations and the dominant energy condition $8 \pi G\left|T_{\alpha \beta}\right| \leqq 8 \pi G T_{00}=1 / 2 R_{i j}^{i j}=0$. The remaining curvature components now vanish because $R_{\alpha \beta}=8 \pi G\left(T_{\alpha \beta}-1 / 2 g_{\alpha \beta} T\right)=0$ $\left(T=T_{\alpha}^{\alpha}\right)$. Thus $N$ is flat along $M$.

\section{The Green's Function}

In this section we will establish the existence of the Green's function for the operator $\mathscr{D}$ by proving Theorem 4.2. The theorem is established through a series of propositions concerning the map $\mathscr{D}: \mathscr{H}_{1, \delta, p} \rightarrow \mathscr{H}_{0, \delta+1, p}$.

Remark. Proposition 5.1, Lemmas 5.2, 5.5 and the estimate of Eq. (5.5) are proved in [4] for similar operators on tensor bundles. The spinor bundle gives no complications, cf. Sect. 2. In fact, Choquet-Bruhat and Christodoulou prove estimates for the weighted Sobolev spaces based on $L_{k}^{2}, k \geqq 0$. Using their results, one can show that statement (ii) of Theorem 4.1 follows from statement (i). Our proof of Theorem 4.2 is self-contained, except for a basic result based on the work of Nirenberg and Walker [9] and Cantor [3].

Proposition 5.1. For $p \geqq 2$ and $0<\delta<2-3 / p$, or $p=2$ and $\delta=-1$, the operators $\nabla$ and $\mathscr{D}$ are bounded linear maps from $\mathscr{H}_{1, \delta, p}$ into $\mathscr{H}_{0, \delta+1, p}$.

Proof. In a local orthonormal frame $\nabla_{i}=\bar{\nabla}_{i}-1 / 2 \sum_{j=1}^{3} h_{i j} e^{0} \cdot e^{j}$. and $\mathscr{D}=$ $\sum_{i=1}^{3} e^{i} \cdot \nabla_{i}$. Thus if $\psi \in \mathscr{H}_{1, \delta, p}$

$$
\left\|\sigma^{1+\delta} \mathscr{D} \psi\right\|_{p} \leqq\left\|\sigma^{1+\delta} \nabla \psi\right\|_{p} \leqq\left\|\sigma^{1+\delta} \bar{\nabla} \psi\right\|_{p}+\left\|\sigma^{1+\delta} \cdot|h| \cdot \psi\right\|_{p}
$$

where $|h|^{2}=\sum_{i, j=1}^{3} h^{i j} h_{i j}$. The proposition follows because $|h| \leqq$ const $\sigma^{-2}$ for asymptotically flat manifolds.

Lemma 5.2. For $p$ and $\delta$ as in Proposition 5.1, there is a continuous embedding 
$\mathscr{H}_{1, \delta, p} \subset \mathscr{H}$. For $\psi \in \mathscr{H}$,

$$
\|\mathscr{D} \psi\|_{2}^{2}=\|\nabla \psi\|_{2}^{2}+\langle\psi, \mathscr{R} \cdot \psi\rangle
$$

with $\mathscr{R} \geqq 0$.

Proof. By Hölder's inequality

and

$$
\|\bar{\nabla} \psi\|_{2}=\left\|\sigma^{-(1+\delta)} \cdot \sigma^{1+\delta} \cdot \bar{\nabla} \psi\right\|_{2} \leqq\left\|\sigma^{-(1+\delta)}\right\|_{2 p / p-2} \cdot\left\|\sigma^{1+\delta} \bar{\nabla} \psi\right\|_{p}
$$

$$
\left\|\sigma^{-1} \psi\right\|_{2}=\|\left(\sigma^{-(1+\delta)} \cdot \sigma^{\delta} \cdot \psi\left\|_{2} \leqq\right\| \sigma^{-(1+\delta)}\left\|_{2 p / p-2} \cdot\right\| \sigma^{\delta} \psi \|_{p} .\right.
$$

The embedding exists because $\sigma^{-(1+\delta)} \in L_{2 p / p-2}$ for $\delta>1 / 2-3 / p$. As for Eq. (5.1), note that by Proposition 5.1 both sides of (5.1) define continuous functionals on $\mathscr{H}$ which are equal on the dense set $C_{0}^{\infty}(M ; S)$ by Eq. (3.2). We have $\mathscr{R} \geqq 0$ from the remark made in Sect. 3.

Proposition 5.3. For $p$ and $\delta$ as in Proposition 5.1, the operator $\mathscr{D}: \mathscr{H}_{1, \delta, p} \rightarrow \mathscr{H}_{0, \delta+1, p}$ is an injection.

Proof. Suppose that $\psi \in \mathscr{H}_{1, \delta, p}$ satisfies $\mathscr{D} \psi=0$. By elliptic regularity (Morrey [8], $\S 6.4) \psi$ is smooth and by Eq. (5.1) $\nabla \psi=0$. Furthermore, $\psi \in \mathscr{H}_{1, \delta, p}$ means that

$$
\int_{M_{l, 2 R}}\left|\sigma^{\delta} \psi\right|^{p}=\int_{S^{2}} \int_{2 R}^{\infty}\left|r^{\delta} \psi\right|^{p}<\infty,
$$

(cf. definitions 4.1 and 4.2) so by Fubini's Theorem $|\psi(x)|$ takes arbitrarily small values along almost every ray to infinity. Now apply Lemma 4.3.

The next two lemmas establish estimates which will be needed to prove the existence of solutions to $\mathscr{D} \psi=\eta$.

Lemma 5.4. For sufficiently large $R$ the following estimate hold for all $\psi \in \mathscr{H}$ :

(a) $\left\|\sigma^{-1} \psi\right\|_{2 ; M_{l, 2 R}}^{2} \leqq 5\|\bar{\nabla} \psi\|_{2 ; M_{l, 2 R}}^{2}$

(b) $\|\bar{\nabla} \psi\|_{2 ; M_{l, 2 R}}^{2} \leqq \frac{6}{5}\|\nabla \psi\|_{2 ; M_{l, 2 R}}^{2}$.

Proof. By continuity it suffices to show this for $\psi \in C_{0}^{\infty}(M ; S)$. Given such a $\psi$ we have, using integration by parts and Hölder's inequality,

$$
\int_{M_{i, 2 R}} r^{-2}|\psi|^{2} d \mu_{3} \leqq 4 \int_{M_{i, 2 R}}|d| \psi||^{2} d \mu_{3} .
$$

Here $r$ is the Euclidean distance in $\mathbb{R}^{3}$ and $d \mu_{3}$ is the Lebesque measure on $\mathbb{R}^{3}$. Because $\bar{\nabla}$ is the connection compatible with the norm $|\psi|^{2}=\langle\psi, \psi\rangle$, we also have $|d| \psi||^{2} \leqq|\bar{\nabla} \psi|^{2}$ (Kato's inequality). Equation (5.2a) now follows by replacing $d \mu_{3}$ by the volume form $\sqrt{g} d \mu_{3}$; this can be done because $g_{i j}=\delta_{i j}+O(1 / r)$ in the asymptotic end $M_{l, R}$.

The inequality (5.2b) is obtained by comparing the covariant derivatives $\bar{\nabla}_{i}$ and $\nabla_{i}=\bar{\nabla}_{i}-1 / 2 h_{i j} e^{0} \cdot e^{j}$. in the end $M_{l, R}$, where $|h| \leqq C_{1} / r^{2}$. In fact, using (5.2a),

$$
\|\nabla \psi\|_{2 ; M_{l, 2 R}} \geqq\|\bar{\nabla} \psi\|_{2 ; M_{l, 2 R}}-\||h| \cdot \psi\|_{2 ; M_{l, 2 R}}
$$


for sufficiently large $R$.

$$
\begin{aligned}
& \geqq\|\bar{\nabla} \psi\|_{2 ; M_{l, 2 R}}-\frac{C_{1}}{R}\left\|\sigma^{-1} \psi\right\|_{2 ; M_{l, 2 R}} \\
& \geqq \frac{5}{6}\|\bar{\nabla} \psi\|_{2 ; M_{i, 2 R}}
\end{aligned}
$$

Lemma 5.5. There exists a constant $0<c=c(R)$ (defined for sufficiently large $R$ ) such that for all $\psi \in \mathscr{H}$,

$$
\|\psi\|_{\mathscr{H}}^{2} \leqq c\|\mathscr{D} \psi\|_{2}^{2} .
$$

Proof. Fix any $R$ large enough that Lemma 5.4 holds and define a cutoff function $\beta=\beta_{R}$ which is identically 1 in each end $M_{l, 3 R}$, which satisfies $0 \leqq \beta \leqq 1$, and which vanishes on $\bar{M}=M-\cup_{l} M_{l, 2 R}$. Given $\psi \in \mathscr{H}$, write $\psi$ as the sum of its interior part $\psi_{\text {in }}=(1-\beta) \psi$ and its exterior part $\psi_{\text {ex }}=\beta \psi$. We shall estimate $\psi_{\text {in }}$ and $\psi_{\mathrm{ex}}$ separately.

First, note that $\psi_{\text {in }}$ has support in $\bar{M}$ and that on $C_{0}^{\infty}(\bar{M} ; S)$ the bilinear form $(\psi, \phi) \rightarrow\langle\nabla \psi, \nabla \phi\rangle$ is positive definite by Lemma 4.3. Standard elliptic theory (Morrey [8], §5.2) then shows that

$$
\left\|\psi_{\text {in }}\right\|_{2}^{2} \leqq c_{2}\left\|\nabla \psi_{\text {in }}\right\|_{2}^{2}
$$

for some constant $c_{2}=c_{2}(R)>0$. This, and the inequality

imply that

$$
\left\|\bar{\nabla} \psi_{\text {in }}\right\|_{2} \leqq\left\|\nabla \psi_{\text {in }}\right\|_{2}+\|h\|_{\infty} \cdot\left\|\psi_{\text {in }}\right\|_{2} \text {, }
$$

$$
\left\|\bar{\nabla} \psi_{\text {in }}\right\|_{2} \leqq c_{3}\left\|\nabla \psi_{\text {in }}\right\|_{2}
$$

for some $c_{3}=c_{3}(R)$.

On the other hand, $\nabla \psi_{\mathrm{ex}}=d \beta \cdot \psi+\beta \cdot \nabla \psi$ with $d \beta=0$ in $\bar{M}$ and $|d \beta| \leqq c_{4} \sigma^{-1}$ outside $\bar{M}$. Combining this with Lemma 5.4 gives

$$
\left\|\nabla \psi_{\mathrm{ex}}\right\|_{2} \leqq c_{5}\|\nabla \psi\|_{2}
$$

where $c_{5}=c_{5}(R)$.

We can now use (5.4) to estimate the total spinor $\psi=\psi_{\text {in }}+\psi_{\text {ex }}$ :

$$
\begin{aligned}
\|\nabla \psi\|_{2}^{2} & =\left\|\nabla \psi_{\text {in }}\right\|_{2}^{2}+\left\|\nabla \psi_{\text {ex }}\right\|_{2}^{2}+2\left\langle\nabla \psi_{\text {in }}, \nabla \psi_{\text {ex }}\right\rangle_{2} \\
& \geqq \frac{1}{2}\left\|\nabla \psi_{\text {in }}\right\|_{2}^{2}+\left\|\nabla \psi_{\text {ex }}\right\|_{2}^{2}-2 c_{5}^{2}\|\nabla \psi\|_{2}^{2} .
\end{aligned}
$$

Rearranging this and substituting in (5.2b) and (5.3) yields

$$
c_{6}\|\bar{\nabla} \psi\|_{2}^{2} \leqq\|\nabla \psi\|_{2}^{2} \text {. }
$$

Finally, using Lemmas 5.2 and $5.4 \mathrm{a}$, we obtain

$$
\|\mathscr{D} \psi\|_{2}^{2} \geqq\|\nabla \psi\|_{2}^{2} \geqq c_{6}\|\bar{\nabla} \psi\|_{2}^{2} \geqq \frac{c_{6}}{2}\|\bar{\nabla} \psi\|_{2}^{2}+\frac{c_{6}}{10}\left\|\sigma^{-1} \psi\right\|_{2}^{2} \geqq c_{7}\|\psi\|_{\mathscr{H}}^{2}
$$

where $c_{7}=c_{7}(R)$ is independent of $\psi \in \mathscr{H}$.

At this point we know (from Propositions 5.1 and 5.3) that $\mathscr{D}: \mathscr{H}_{1, \delta, p} \rightarrow \mathscr{H}_{0, \delta+1, p}$ 
is continuous and injective. We shall next demonstrate that it is surjective. This is done in two steps. We first prove that, given $\eta \in \mathscr{H}_{0 . \delta+1, p}$, there exists a unique $\psi \in \mathscr{H}$ with $\mathscr{D} \psi=\eta$. The second step establishes that $\psi \in \mathscr{H}_{1, \delta, p}$ by proving an a priori estimate for $\|\psi\|_{1, \delta, p}$ in terms of $\|\eta\|_{0, \delta+1, p}$.

Proposition 5.6. For each $\eta \in C_{0}^{\infty}(M ; S)$ there exists a unique $u \in \mathscr{H}$ such that $\mathscr{D}^{2} u=\eta$. Furthermore, $\psi=\mathscr{D} u \in \mathscr{H}$ and

$$
\|\psi\|_{\mathscr{H}}^{2} \leqq c\|\eta\|_{2}^{2}
$$

where $c$ is the constant of Lemma 5.5.

Proof. The equation $\mathscr{D}^{2} u=\mathscr{D}^{*} \mathscr{D} u=\eta$ is formally the variational equation of the functional

$$
\mathscr{F}(u)=\frac{1}{2}\|\mathscr{D} u\|_{2}^{2}+\langle u, \eta\rangle_{2} .
$$

It follows from Proposition 5.1 that for $\eta \in C_{0}^{\infty}(M ; S) \mathscr{F}$ is a $C^{\infty}$ functional on the Hilbert space $\mathscr{H}$. The functional $u \mapsto\langle u, \eta\rangle_{2}$ is continuous and linear and $u \mapsto\|\mathscr{D} u\|_{2}^{2}$ is strictly convex; hence $\mathscr{F}$ is strictly convex and weakly lowersemicontinuous. Lemma 5.5 implies the lower bound

$$
|\mathscr{F}(u)| \geqq \frac{1}{2 c}\|u\|_{\mathscr{H}}^{2}-\|u\|_{\mathscr{H}} \cdot\|\sigma \eta\|_{2}
$$

Hence, by the calculus of variations (Vainberg [14]), the functional $\mathscr{F}(u)$ has a unique critical point $u \in \mathscr{H}$ which is an absolute minimum in $\mathscr{H}$, and $u$ is a weak solution of the variational equation. Standard elliptic regularity arguments imply that $u$ is smooth. Set $\psi=\mathscr{D} u \in \mathscr{H}$. Then $\mathscr{D} \psi=\eta$ and Lemma 5.5 gives the stated inequality.

Proposition 5.7. For each $\eta \in C_{0}^{\infty}(M ; S)$ there exists a unique $\psi \in \mathscr{H}$ with $\mathscr{D} \psi=\eta$. For $p$ and $\delta$ as in Proposition 5.1.

$$
\|\psi\|_{1, \delta, p} \leqq c(\delta, p)\|\eta\|_{0, \delta+1, p} .
$$

Proof. Let $u$ be the solution $\mathscr{D}^{2} u=\eta$ obtained from Proposition 5.6 and set $\psi=\mathscr{D} u \in \mathscr{H}$. Then $\psi$ satisfies $\mathscr{D} \psi=\eta$.

We again separate $\psi$ into its interior part $\psi_{\text {in }}=(1-\beta) \psi$ and its exterior part $\psi_{\mathrm{ex}}=\beta \psi$ and estimate each part separately.

The estimates for $\psi_{\text {in }}$ are straightforward: since $\psi_{\text {in }}$ has support in the bounded domain $\bar{M}$, standard elliptic estimates (Morrey [8], §6.2) imply that

$$
\mid \bar{\nabla} \psi_{\text {in }}\left\|_{p ; \bar{M}}+\right\| \psi_{\text {in }}\left\|_{p ; \bar{M}} \leqq c_{8}\right\| \eta \|_{\mid p ; M} .
$$

We shall estimate $\psi_{\text {ex }}$ on each asymptotic end $M_{l} \subset \mathbb{B}^{3}$ by extending $\psi$ and $\mathscr{D}$ to all of $\mathbb{R}^{3}$. This is done as follows. Fix an end $M_{l}$ with defining diffeomorphism $\Phi_{l}: \mathbb{R}^{3} \backslash K_{l} \rightarrow M_{l}$. Let $R_{0}>0$ be such that $M_{l, 2 / 3 R_{0}} \subset M_{l}$. As noted in Sect. $2, \Phi_{l}^{*} S$ is the trivial spin bundle over $M_{l} \subset \mathbb{R}^{3}$, so we can extend $\psi_{\text {ex }}$ and $\eta$ to spinors on $\mathbb{R}^{3}$. For each $R>R_{0}$ extend the metric $g_{i j}$ to a metric $g_{i j}^{R}$ on $\mathbb{R}^{3}$ in such a way that $g^{R}=g$ on $M_{l, R}$ by setting $g_{i j}^{R}(x)=\delta_{i j}+\beta(3 x)\left(g_{i j}(x)-\delta_{i j}\right)$. For each $R>R_{0}$, 
let $\mathscr{D}_{R}$ be the operator on $C^{\infty}\left(\mathbb{R}^{3} ; \mathbb{R}^{3} \times S\right)$ defined by

$$
\mathscr{D}_{R}=\sum_{i=1}^{3} e_{R}^{i} \cdot \bar{\nabla}_{i}^{R}-\frac{1}{2} \beta(3 x) h_{i j}(x) e^{0} .
$$

where $\bar{\nabla}_{i}^{R}=\partial_{i}+\beta(3 x) \Gamma_{i}(x),\left.\Gamma_{i}\right|_{M_{l}}$ is the spin connection on $\left.S\right|_{M_{l}}$ of the metric $g_{i j},\left\{e_{R}^{i}\right\}$ is a $g^{R}$-orthonormal coframe, and $e^{0}$. is the standard hermitian structure on $\mathbb{R}^{3} \times S$. With these definitions we have $\mathscr{D}_{0}=\sum_{i=1}^{3} d x^{i} \partial_{i}$, while $\mathscr{D}_{R}=\mathscr{D}$ on sections of $\Phi_{l}^{*} S$ with support in $M_{l, R}$.

Note that the asymptotic decay assumptions imply that given $\varepsilon>0$, there exists $R_{0}<R<\infty$ such that

and

$$
\sup _{x \in \mathbb{R}^{3}}\left|e_{R}^{i}-d x^{i}\right|<\varepsilon
$$

$$
\sup _{x \in \mathbb{R}^{3}} \sigma \cdot\left(\left|\Gamma_{i}\right|+\left|h_{i j}\right|\right) \cdot \beta(3 x)<\varepsilon .
$$

This fact allows us to utilize a theorem due to M. Cantor (based on the work of Nirenberg and Walker [9].)

Theorem 5.8. (Cantor [3], Theorem 1.3.). Under the assumptions on the asymptotic decay of $g_{i j}$ and $h_{i j}$ stated in Sect. 1 , one can choose $R<\infty$, sufficiently large such that when $p \geqq 2$ and $0<\delta<2-3 / p$,

$$
\mathscr{D}_{\mathbb{R}}: \mathscr{H}_{1, \delta, p}\left(\mathbb{R}^{3}, S\right) \rightarrow \mathscr{H}_{0, \delta+1, p}\left(\mathbb{R}^{3} ; S\right)
$$

is an isomorphism with bounded inverse.

We can now complete the proof of Proposition 5.7. On $\mathbb{R}^{3}, \psi_{\mathrm{ex}}=\beta \psi$ satisfies

$$
\mathscr{D}_{R}\left(\psi_{\mathrm{ex}}\right)=d \beta \cdot \psi+\beta \cdot \eta \text {. }
$$

Since $\mathscr{H}_{1, \delta, p}\left(\mathbb{R}^{3} ; S\right) \subset \mathscr{H}\left(\mathbb{R}^{3}, S\right)$ and $\psi_{\text {ex }} \in \mathscr{H}\left(\mathbb{R}^{3} ; S\right)$, Theorem 5.8 is applicable; it gives

$$
\left\|\psi_{\mathrm{ex}}\right\|_{1, \delta, p} \leqq c(\delta, p)\|\beta \cdot \eta\|_{0, \delta+1, p}
$$

for $p \geqq 2$ and $0<\delta<2-3 / p$. The norms in (5.8) are for the Euclidean metric on $\mathbb{R}^{3}$ but, because $g_{i j}=\delta_{i j}+O(1 / r)$ and $\left|\partial_{k} g_{i j}\right|=O\left(1 / r^{2}\right)$ in $\mathbb{R}^{3}-B_{R}$, these norms are equivalent to the norms induced by $g_{i j}$.

We now have (5.8) on each end and (5.6) in the interior; together these give (5.5) for $p \geqq 2$ and $0<\delta<2-3 / p$. The case $p=2, \delta=-1$ was obtained in Proposition 5.6.

Proposition 5.9. Let $p$ and $\delta$ be as in Proposition 5.1. Then $\mathscr{D}: \mathscr{H}_{1, \delta, p} \rightarrow \mathscr{H}_{0, \delta+1, p}$ is a surjection. If $\eta \in \mathscr{H}_{0 . \delta+1, p}$ and $\psi \in \mathscr{H}_{1, \delta, p}$ with $\mathscr{D} \psi=\eta$, then the a priori bound (5.5) holds.

Proof. Given $\eta \in \mathscr{H}_{0, \delta+1, p}$, choose a sequence $\left\{\eta_{i}\right\} \in C_{0}^{\infty}(M ; S)$ which coverges strongly to $\eta$. For each $i$, let $\psi_{i} \in \mathscr{H}$ be the solution of $\mathscr{D} \psi_{i}=\eta_{i}$ found in Proposition 5.7. Since $\mathscr{D}\left(\psi_{i}-\psi_{j}\right)=\eta_{i}-\eta_{j},(5.5)$ implies that $\left\{\psi_{i}\right\}$ is a Cauchy sequence in $\mathscr{H}_{1, \delta, p}$. 
Let $\psi$ denote its limit. Then

$$
\|\mathscr{D} \psi-\eta\|_{0, \delta+1, p} \leqq\left\|\psi-\psi_{i}\right\|_{1, \delta, p}+\left\|\eta-\eta_{i}\right\|_{0, \delta+1, p}
$$

for all $i$, so $\mathscr{D} \psi=\eta$. The a priori bound (5.5) follows similarly.

Propositions 5.1, 5.3 and 5.9 show that $\mathscr{D}: \mathscr{H}_{1, \delta, p} \rightarrow \mathscr{H}_{0, \delta+1, p}$ is an isomorphism and that $\mathscr{D}$ and $\mathscr{D}^{-1}$ are bounded linear maps. Also, if $\mathscr{D} \psi=\eta$ with $\eta \in C^{\infty}(M ; S)$, then $\psi \in C^{\infty}(M ; S)$ by elliptic regularity. This completes the proof of Theorem 4.2.

Acknowledgements. We thank Professors R. Bott, A. Jaffe, and S.-T. Yau for their suggestions and encouragement.

\section{References}

1. Arnowitt, R., Deser, S., Misner, C.: Phys. Rev. 118, 1100 (1960)

2. Arnowitt, R., Deser, S., Misner, C.: Coordinate invariance and energy expressions in general relativity. Phys. Rev. 122, 997-1006 (1961)

3. Cantor, M.: Some problems of global analysis on asymptotically simple manifolds. Compositio Math. 38, 3-35 (1979)

4. Choquet-Bruhat. Y., Christodoulou, D.: Elliptic systems in $H_{s, \delta}$ spaces on manifolds which are Euclidean at infinity. Acta. Math. 146, 124-150 (1981)

5. Geroch, R.: General relativity. Proc. Symp. Pure Math. 27, 401-414 (1975)

6. Hawking, S., Ellis, G.: The large scale structure of space-time. Cambridge: Cambridge University Press 1973

7. Hitchen, N.: Harmonic spinors. Adv. Math. 14, 1-55 (1974)

8. Morrey, C. B.: Multiple integrals in the calculus of variations. New York: Springer 1966

9. Nirenberg, L., Walker, H.: The null spaces of elliptic partial differential operators in $\mathbb{R}^{N}$. J. Math. Anal. App. 47, 271-301 (1973)

10. Schoen, R., Yau, S.-T.: On the proof of the positive mass conjecture in general relativity. Commun. Math. Phys. 65, 45-76 (1976).

11. Schoen, R., Yau, S.-T.: Positivity of the total mass in a general space-time. Phys. Rev. Lett. 43, 14571459 (1979)

12. Schoen, R., Yau, S.-T.: Proof of the positive mass theorem II. Commun. Math. Phys. 79, 231 (1981)

13. Schoen, R., Yau, S.-T.: The energy and the linear momentum of space-times in general relativity. Commun. Math. Phys. 79, 47 (1981).

14. Vainberg, M.: Variational methods and the method of monotone operators in the theory of nonlinear equations. New York: Wiley 1973

15. Witten, E.: A simple proof of the positive energy theorem. Commun. Math. Phys. 80, 381-402.(1931)

Communicated by A. Jaffe

Received September 23, 1981 